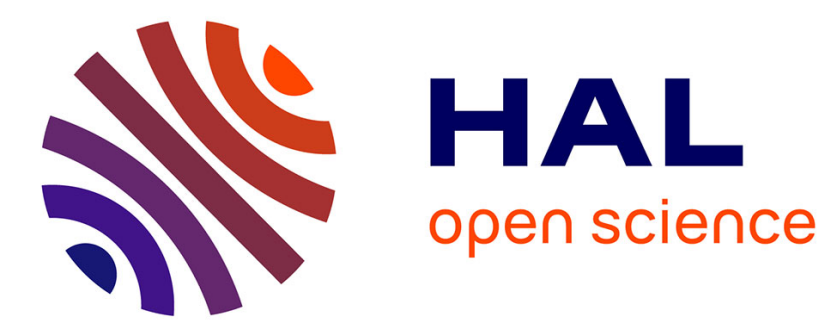

\title{
Dynamic System Performance of SISO, MISO and MIMO Alamouti Schemes
}

Dorra Ben Cheikh, Jean-Marc Kélif, Marceau Coupechoux, Philippe

Godlewski

\section{- To cite this version:}

Dorra Ben Cheikh, Jean-Marc Kélif, Marceau Coupechoux, Philippe Godlewski. Dynamic System Performance of SISO, MISO and MIMO Alamouti Schemes. IEEE Sarnoff Symposium, May 2011, Princeton, United States. pp.1-5. hal-00717680

\section{HAL Id: hal-00717680 https://hal-imt.archives-ouvertes.fr/hal-00717680}

Submitted on 13 Jul 2012

HAL is a multi-disciplinary open access archive for the deposit and dissemination of scientific research documents, whether they are published or not. The documents may come from teaching and research institutions in France or abroad, or from public or private research centers.
L'archive ouverte pluridisciplinaire HAL, est destinée au dépôt et à la diffusion de documents scientifiques de niveau recherche, publiés ou non, émanant des établissements d'enseignement et de recherche français ou étrangers, des laboratoires publics ou privés. 


\title{
Dynamic System Performance of SISO, MISO and MIMO Alamouti Schemes
}

\author{
Dorra Ben Cheikh Battikh ${ }^{\dagger \star}$, Jean-Marc Kelif ${ }^{\star}$, Marceau Coupechoux ${ }^{\dagger}$ and Philippe Godlewski ${ }^{\dagger}$ \\ * Orange Labs, Issy-les-moulineaux, France \\ Email: \{dorra.bencheikh, jeanmarc.kelif\}@orange-ftgroup.com \\ ${ }^{\dagger}$ TELECOM Paris Tech \& CNRS LTCI, Paris, France \\ Email: $\{$ bencheikh, coupecho, godlewski\}@enst.fr
}

\begin{abstract}
In this paper, the performance of a SISO system, a $2 \times 1$ MISO Alamouti transmission system and a $2 \times N$ MIMO Alamouti transmission with maximum ratio combining (MRC) receiver system is studied in terms of mean flow throughput per user. A dynamic study (dynamic number of active users) is derived considering the downlink Rayleigh channel in a multicellular system. Two assumptions are considered: admission control based on a maximum number of users or no admission control. The studied cell is divided into concentric rings. Analytical expressions of the mean flow throughput of a user in each ring are proposed. A comparison between the performance of the different systems is presented and discussed.
\end{abstract}

\section{INTRODUCTION}

MIMO systems have been deeply investigated during the last years. They have been proven to achieve better performance in terms of capacity through the multiplexing gain [1] and in terms of reliability through to the diversity gain [2]. A tradeoff between these two promised gains have been established [2]. Space-time codes [3] was introduced as a technique permitting to achieve this tradeoff. In this context, the Alamouti code was proposed in [4] as a space-time code meeting the diversity multiplexing tradeoff for a $2 \times 1$ MISO system. Many other techniques have been proposed to exploit antennas diversity. The maximum ratio combining (MRC) [5] is a reception technique achieving the maximum receive diversity. All these techniques have been studied for a constant traffic demand.

In the present work, we will consider a dynamic traffic demand. This assumption has been considered in [6], where authors derived an analytical performance study for downlink data channels with dynamic traffic demand in a single cell system considering path loss effect. In [7], the same authors, derived upper and lower bound of the flow throughput in a multi-cell SISO system taking into consideration again the path loss effect. In [8], the author studied the influence of the proportional fair channel-aware scheduling on the mean user throughput when considering a dynamic set of users.

In this paper, we extend these results by deriving an analytical performance comparison between SISO, $2 \times 1$ MISO Alamouti and $2 \times N$ Alamouti with MRC receiver systems in a dynamic traffic demand context for downlink data channels. We consider a time-shared system where each user transmits in a different time slot. The cell being divided into concentric rings, we compare the flow throughput of each system in each ring with or without admission control.

The reminder of the paper is organized as follows. In section II, we describe the three system models. In section III, we introduce the fluid model allowing us to obtain an approximation of SINR expression simplifying the analytical study in a multi-cellular environment. Section IV presents the dynamic traffic model used in our analysis and the analytical expressions of the mean flow throughput. In section V, systems performances are presented and discussed. Finally, section VI concludes the paper.

\section{SyStem MODELS}

\section{A. SISO System}

Consider a SISO multi-cellular system and consider a single user per cell. The base-station (BS) common transmit power is denoted $P_{T}$. The signal received by a user served by the BS 0 and interfered by $B$ BSs is given by:

$$
y=\sqrt{P_{0}} h_{0} x_{0}+\sum_{j=1}^{B} \sqrt{P_{j}} h_{j} x_{j}+n,
$$

where $h_{j}$ is the flat fading Rayleigh channel gain between the BS $j$ and the considered user, $x_{j}$ is the signal transmitted by $\mathrm{BS} j$ and $n$ is the additive white Gaussian noise. $P_{j}=K P_{T} d_{j}^{-\eta}$ is the power received by the user from BS $j$ including the path loss term, where $K$ is a constant, $d_{j}$ is the distance between BS $j$ and the considered user and $\eta>2$ is the path-loss exponent.

The output SINR at the considered user can be written as:

$$
\gamma_{S I S O}=\frac{P_{0}\left|h_{0}\right|^{2}}{\sum_{j=1}^{B} P_{j}\left|h_{j}\right|^{2}+\sigma_{n}^{2}} .
$$

In an interference limited system the SINR can be approximated by:

$$
\gamma_{S I S O} \approx \frac{X_{S I S O}}{Y_{S I S O}}
$$

where

$$
X_{S I S O}=P_{0}\left|h_{0}\right|^{2}, \quad \text { and } \quad Y_{S I S O}=\sum_{j=1}^{B} P_{j}\left|h_{j}\right|^{2} .
$$


Since we considered Rayleigh flat fading, it can be easily noticed that $X_{S I S O}$ is exponentially distributed with probability density function (PDF):

$$
f_{X_{S I S O}}(x)=\frac{1}{P_{0}} e^{-\frac{x}{P_{0}}},
$$

Using the central limit theorem for causal function [9], the PDF of $Y_{S I S O}$ can be approximated by a Gamma distribution and is given by:

$$
f_{Y_{S I S O}}(y)=\frac{y^{\nu-1}}{\Gamma(\nu) \delta^{\nu}} e^{-\frac{y}{\delta}}
$$

where $\nu=\frac{\mathrm{E}(y)^{2}}{\operatorname{var}(y)}$ and $\delta=\frac{\operatorname{var}(y)}{\mathrm{E}(y)}$ are given by [10]:

$$
\nu=\frac{1}{2} \frac{\left(\sum_{j=1}^{B} P_{j}\right)^{2}}{\sum_{j=1}^{B} P_{j}^{2}}, \delta=2 \frac{\sum_{j=1}^{B} P_{j}^{2}}{\sum_{j=1}^{B} P_{j}} .
$$

Using the PDFs (1) and (2) we can derive the PDF of the SINR for a SISO system as:

$$
\begin{aligned}
f_{\gamma_{S I S O}}(\gamma) & =\int_{0}^{\infty} f_{X_{S I S O}}(\gamma y) f_{Y_{S I S O}}(y) y d y \\
& =\frac{P_{0}^{\nu} \delta \nu}{\left(\delta \gamma+P_{0}\right)^{\nu+1}} .
\end{aligned}
$$

\section{B. MISO Alamouti System}

Consider, now, a MISO Alamouti multi-cellular system where each BS is equipped with two antennas and the users' equipment with a single antenna. All BSs use the Alamouti code to transmit their data. At the receiver the signal is multiplied by the Alamouti receiver (see e.g. [11]).

By pre-multiplying the received signal by the channel transpose conjugate of the channel $\mathbf{H}_{0}$, the signal at the receiver becomes:

$$
\begin{aligned}
\overline{\mathbf{H}}_{0} \mathbf{y} & =\sqrt{\frac{P_{0}}{2}}\left[\begin{array}{cc}
\left|h_{1,0}\right|^{2}+\left|h_{2,0}\right|^{2} & 0 \\
0 & \left|h_{1,0}\right|^{2}+\left|h_{2,0}\right|^{2}
\end{array}\right] \mathbf{x}_{0} \\
& +\sum_{j=1}^{B} \sqrt{\frac{P_{j}}{2}}\left[\begin{array}{cc}
h_{1,0} & h_{2,0} \\
h_{2,0}^{*} & -h_{1,0}^{*}
\end{array}\right]^{H}\left[\begin{array}{cc}
h_{1, j} & h_{2, j} \\
h_{2, j}^{*} & -h_{1, j}^{*}
\end{array}\right] \mathbf{x}_{j} \\
& +\left[\begin{array}{cc}
h_{1,0} & h_{2,0} \\
h_{2,0}^{*} & -h_{1,0}^{*}
\end{array}\right]^{H} \mathbf{n} .
\end{aligned}
$$

where $\mathbf{x}_{\mathbf{j}}$ is the symbols vector transmitted by BS $j ; h_{i, j}$ is the flat fading Rayleigh channel gain between the antenna $i$ of BS $j$ and its user (the flat fading is assumed to be quasi-static over the two channel use periods); and $\mathbf{n}$ is the additive white Gaussian noise vector with covariance matrix $\sigma_{n}^{2} \mathbf{I} . P_{j}$ is the received power from the $j^{\text {th }} \mathrm{BS}$ ( $P_{0}$ is the power received from the serving BS) including path-loss.

The output SINR per stream is, hence, given by:

$$
\gamma_{\text {Alam }}=\frac{\frac{P_{0}}{2}\left(\left|h_{1,0}\right|^{2}+\left|h_{2,0}\right|^{2}\right)}{\sum_{j=1}^{B} \frac{P_{j}}{2} \frac{\left|h_{1,0}^{*} h_{1, j}+h_{2,0} h_{2, j}^{*}\right|^{2}+\left|h_{1,0}^{*} h_{2, j}-h_{2,0} h_{1, j}^{*}\right|^{2}}{\left|h_{1,0}\right|^{2}+\left|h_{2,0}\right|^{2}}+\sigma_{n}^{2}}
$$

In an interference limited system, the SINR of the MISO Alamouti system can be again approximated as: $\gamma_{\text {Alam }} \approx$ $X_{\text {Alam }} / Y_{\text {Alam }}$, where

$$
\begin{aligned}
X_{\text {Alam }} & =\frac{P_{0}}{2}\left(\left|h_{1,0}\right|^{2}+\left|h_{2,0}\right|^{2}\right), \\
Y_{\text {Alam }} & =\sum_{j=1}^{B} \frac{P_{j}}{2} \frac{\left|h_{1,0}^{*} h_{1, j}+h_{2,0} h_{2, j}^{*}\right|^{2}+\left|h_{1,0}^{*} h_{2, j}-h_{2,0} h_{1, j}^{*}\right|^{2}}{\left|h_{1,0}\right|^{2}+\left|h_{2,0}\right|^{2}}
\end{aligned}
$$

From [10], the PDF of $X_{\text {Alam }}$ is given by:

$$
f_{X_{\text {Alam }}}(x)=\frac{4 x}{P_{0}^{2}} e^{-\frac{2 x}{P_{0}}} .
$$

and the PDF of $Y_{\text {Alam }}$ is approximated by:

$$
f_{Y_{\text {Alam }}}(y)=\frac{y^{\alpha-1} \exp \left(-\frac{y}{\beta}\right)}{\Gamma(\alpha) \beta^{\alpha}},
$$

where $\alpha=\frac{\mathrm{E}[Y]^{2}}{\operatorname{var}(Y)}$ and $\beta=\frac{\operatorname{var}(Y)}{\mathrm{E}[Y]}$. $\alpha$ and $\beta$ are given by [10]:

$$
\alpha=\frac{2}{1+\epsilon} \frac{\left(\sum_{j=1}^{B} P_{j}\right)^{2}}{\sum_{j=1}^{B} P_{j}^{2}}, \beta=\frac{1+\epsilon}{2} \frac{\sum_{j=1}^{B} P_{j}^{2}}{\sum_{j=1}^{B} P_{j}},
$$

and $\epsilon=-0.0187$. Following the same method as for (4), the distribution of $\gamma_{\text {Alam }}$ is given by:

$$
f_{\gamma_{\text {Alam }}}(z)=4 \beta^{2} P_{0}^{\alpha} \alpha(\alpha+1) \frac{z}{\left(2 \beta z+P_{0}\right)^{\alpha+2}}
$$

\section{MIMO Alamouti System with MRC Receiver}

We now assume that BSs are equipped with 2 antennas and user equipments with $N$ antennas. An MRC receiver combines the received signals from the $N$ antennas. In a interference limited environment, the SINR can still be written as: $\gamma_{M R C} \approx$ $X_{M R C} / Y_{M R C}$, where

$$
\begin{aligned}
X_{M R C} & =\frac{P_{0}}{2} \sum_{n=1}^{N}\left(\left|h_{n, 1,0}\right|^{2}+\left|h_{n, 2,0}\right|^{2}\right), \\
Y_{M R C} & =\sum_{j=1}^{B} \frac{P_{j}}{2}\left(\frac{\left|\sum_{n=1}^{N} h_{n, 1,0}^{*} h_{n, 1, j}+h_{n, 2,0} h_{n, 2, j}^{*}\right|^{2}}{\sum_{n=1}^{N}\left(\left|h_{n, 1,0}\right|^{2}+\left|h_{n, 2,0}\right|^{2}\right)}\right. \\
& \left.+\frac{\left|\sum_{n=1}^{N} h_{n, 1,0}^{*} h_{n, 2, j}-h_{n, 2,0} h_{n, 1, j}^{*}\right|^{2}}{\sum_{n=1}^{N}\left(\left|h_{n, 1,0}\right|^{2}+\left|h_{n, 2,0}\right|^{2}\right)}\right),
\end{aligned}
$$

and $h_{n, i, j}$ is the flat fading Rayleigh channel gain between the $i^{t h}$ antenna of BS $j$ and the $n^{t h}$ antenna of the served user. From [12], the distributions of $X_{M R C}$ and $Y_{M R C}$ are given by:

$$
\begin{aligned}
f_{X_{M R C}}(x) & =\frac{x^{2 N-1}}{\left(\frac{P_{0}}{2}\right)^{2 N}(2 N-1) !} e^{-\frac{2 x}{P_{0}}}, \\
f_{Y_{M R C}}(y) & =\frac{y^{v-1} \exp \left(-\frac{y}{\tau}\right)}{\Gamma(v) \tau^{v}}
\end{aligned}
$$

where $v$ and $\tau$ are given by:

$$
v=\frac{2}{1+\zeta} \frac{\left(\sum_{j=1}^{B} P_{j}\right)^{2}}{\sum_{j=1}^{B} P_{j}^{2}}, \quad \tau=\frac{(1+\zeta)}{2} \frac{\sum_{j=1}^{B} P_{j}^{2}}{\sum_{j=1}^{B} P_{j}} .
$$


and $\zeta=0.0043$.

The distribution of $\gamma_{M R C}$ can be calculated as for (4), and is given by:

$$
f_{\gamma_{M R C}}(u)=\frac{\Gamma(2 N+v)\left(\frac{P_{0}}{2}\right)^{v}}{(2 N-1) ! \Gamma(v) \tau^{v}} \frac{u^{2 N-1}}{\left(u+\frac{P_{0}}{2 \tau}\right)^{2 N+v}} .
$$

Note that setting $N=1$ in (9) yields (7).

\section{Fluid Model Approximation}

To derive an outage probability expression depending only on the distance between the considered user and the serving BS we use the fluid model approximation described in [13]. The fluid model concept consists in replacing a fixed number of BSs by an equivalent continuum characterized by a given density. Let us define:

$$
g(\eta)=\sum_{j=1}^{B} d_{j}^{-\eta} .
$$

For an infinite homogeneous network and a BS density $\rho_{B S}$, the fluid model permits to write $g(\eta)$ as:

$$
g(\eta)=\frac{2 \pi \rho_{B S}}{\eta-2}\left(2 R_{c}-r\right)^{2-\eta}
$$

where $R_{c}$ is the considered cell radius and $r$ is the distance between the considered user and its serving BS.

In terms of $g(\eta)$, the parameters of the outage probability of the multi-cellular SISO system given by $\nu$ and $\delta$ (3) can be written as:

$$
\nu=\frac{1}{2} \frac{g(\eta)^{2}}{g(2 \eta)}, \delta=2 K P_{T} \frac{g(2 \eta)}{g(\eta)} .
$$

Similarly, the parameters of the MISO Alamouti system $\alpha$ and $\beta$ (6) are given by:

$$
\alpha=\frac{2}{1+\epsilon} \frac{g(\eta)^{2}}{g(2 \eta)}, \beta=\frac{1+\epsilon}{2} K P_{T} \frac{g(2 \eta)}{g(\eta)},
$$

and the parameters of the MIMO Alamouti with MRC receiver system $v$ and $\tau$ (8) are given by:

$$
v=\frac{2}{1+\zeta} \frac{g(\eta)^{2}}{g(2 \eta)}, \quad \tau=\frac{1+\zeta}{2} K P_{T} \frac{g(2 \eta)}{g(\eta)},
$$

\section{Dynamic Traffic Study}

We now assume a dynamic system where users randomly enter the network, download a file and go out of the system. We are interested in the user throughput.

\section{A. Traffic Model}

We consider a cellular network with a time-shared downlink. The scheduler is fair in slots: when there are $n$ active users, each one receives $1 / n$ proportion of time for data transmission. We assume a uniform traffic demand in the cell, flow sizes are independent and identically distributed with a mean flow size equal to $V$. The data flow arrival process is Poisson with an intensity per surface area $\lambda$.

Let us divide the cell in $K^{\prime}$ concentric rings around BS 0 defined by ranges $R_{k}, k \in\left\{1, \ldots, K^{\prime}\right\}, R_{K^{\prime}}=R_{c}$ and
$R_{0}=0$. The arrival rate in ring $k$ is $\lambda_{k}=p_{k} \lambda \pi R_{c}^{2}$, with $p_{k}=\left(R_{k}^{2}-R_{k-1}^{2}\right) / R_{c}^{2}$. The service time in ring $k$ is $V / c_{k}$, where $c_{k}$ is the average throughput in ring $k$. So the load generated by ring $k$ is $\rho_{k}=\lambda \pi R_{c}^{2} V p_{k} / c_{k}$ and the cell load is:

$$
\rho=\sum_{k=1}^{K^{\prime}} \rho_{k}=\lambda \pi R_{c}^{2} V \sum_{k=1}^{K^{\prime}} \frac{p_{k}}{c_{k}}=\frac{\lambda \pi R_{c}^{2} V}{C},
$$

where

$$
C=\left(\sum_{k}^{K^{\prime}} \frac{p_{k}}{c_{k}}\right)^{-1}
$$

is the cell capacity.

With the model proposed in the previous section, we are able to compute $c_{k}$ :

$$
c_{k}=\int_{R_{k-1}}^{R_{k}} \frac{2 r D(r) d r}{R_{k}^{2}-R_{k-1}^{2}},
$$

where $D(r)$ is the mean achievable rate at distance $r$ and is given by:

$$
D(r)=W \int_{0}^{\infty} \log _{2}(1+\gamma(r)) f_{\gamma}(\gamma(r)) d \gamma .
$$

In case of no admission control, the flow throughput of a user in the ring $k$ is then given by [6]:

$$
\varphi_{k}=c_{k}(1-\rho) .
$$

We now consider an admission control based on a maximum number, $m$ of active users. This number of users can be chosen in order to ensure a minimum rate $c_{\min }$, hence,

$$
m=\left\lfloor\frac{C}{c_{\min }}\right\rfloor \text {. }
$$

In this case, the flow throughput in ring $k$ is given by [6]:

$$
\varphi_{k}=c_{k} \frac{(1-\rho)\left(1-\rho^{m}\right)}{1-(m+1) \rho^{m}+m \rho^{m+1}} .
$$

We now derive the mean achievable rate $D(r)$ for each considered system.

\section{B. SISO System}

The mean achievable at rate a distance $r$ for a SISO system, is given by:

$$
\begin{aligned}
D(r) & =\frac{W}{\log (2)} \int_{0}^{\infty} \log (1+\gamma(r)) f_{\gamma_{S I S O}}(\gamma(r)) d \gamma, \\
& =\frac{W}{\log (2)}\left(\frac{P_{0}}{\delta}\right)^{\nu} \int_{0}^{\infty}(1+\gamma)^{-1}\left(\gamma+\frac{P_{0}}{\delta}\right)^{-\nu} d \gamma, \\
& =\frac{W}{\log (2)} \frac{P_{0}}{\delta \nu}{ }_{2} F_{1}\left(1,1 ; \nu+1 ; 1-\frac{P_{0}}{\delta}\right) .
\end{aligned}
$$

where ${ }_{2} F_{1}$ is the hypergeometric function, and $\nu(r)$ and $\delta(r)$ are given by (10). 


\section{MISO Alamouti}

The mean achievable at rate a distance $r$ for the MISO Alamouti system, is given by:

$$
\begin{gathered}
D_{M I S O}(r)=\frac{W}{\log (2)} \int_{0}^{\infty} \log (1+\gamma) f_{\gamma_{\text {Alam }}}(\gamma) d \gamma \\
=\frac{W}{\log (2)} \frac{P_{0}}{2 \beta} \int_{0}^{\infty} \gamma \log (1+\gamma)\left(\gamma+\frac{P_{0}}{2 \beta}\right)^{-(\alpha+2)} d \gamma .
\end{gathered}
$$

After integration by parts, $D(r)$ can be written as:

$$
\begin{gathered}
D_{M I S O}(r)= \\
\frac{W}{\log (2)} \frac{P_{0}}{2 \beta}\left(\frac{1}{\alpha}{ }_{2} F_{1}\left(1,1 ; \alpha+1 ; 1-\frac{P_{0}}{2 \beta}\right)\right. \\
\left.+\frac{1}{\alpha+1}{ }_{2} F_{1}\left(1,2 ; \alpha+2 ; 1-\frac{P_{0}}{2 \beta}\right)\right) .
\end{gathered}
$$

\section{MIMO Alamouti with MRC Receiver}

At a distance $r$, the mean achievable rate for a MIMO Alamouti coded system with an MRC receiver can be written as:

$$
\begin{gathered}
D_{M I M O}(r)=\frac{W}{\log (2)} \int_{0}^{\infty} \log (1+\gamma) f_{\gamma_{M R C}}(\gamma) d \gamma \\
=\frac{W}{\log (2)} \frac{\Gamma(2 N+v)\left(\frac{P_{0}}{2}\right)^{v}}{(2 N-1) ! \Gamma(v) \tau^{v}} \times \int_{0}^{\infty} \log (1+\gamma) \frac{\gamma^{2 N-1}}{\left(\gamma+\frac{P_{0}}{2 \tau}\right)^{2 N+v}} d \gamma .
\end{gathered}
$$

\section{SYSTEMS PERFORMANCE}

In this section we will study the performance of the three previously introduced systems with and without admission control.

\section{A. Assumptions}

In all case, we consider a mean flow size of $V=2$ Mbits, a channel bandwidth of $5 \mathrm{MHz}$ and a cell radius $R_{c}=1 \mathrm{Km}$. The cell is divided into five concentric rings characterized by radii $0.2,0.4,0.6,0.8$ and $1 \mathrm{Km}$.

Average throughputs $c_{k}$ are obtained using Mathematica ${ }^{\mathrm{TM}}$ from (15). As suggested in [14] for LTE, the Shannon formula has been multiplied by a loss factor of 0.6 in order to take into account imperfect rate adaptation. Moreover, in all calculations, we consider that users are located at distances higher than $0.05 \mathrm{Km}$ from the BS.

\section{B. No Admission Control}

Based on the equation (17) we calculate the mean flow throughput in each ring for SISO, the MISO Alamouti and the MIMO Alamouti with MRC systems. Fig. 1 presents a comparison between the mean flow throughput of the SISO system and the $2 \times 1$ Alamouti system as a function of the cell load $\rho$. In terms of achievable throughputs, MISO Alamouti slightly outperforms SISO, especially in the inner rings. Performances are lower at cell edge. Note that our analysis does not focus on the outage probability (see [10] for a detailed study on this question), which Alamouti is clearly advantageous for.

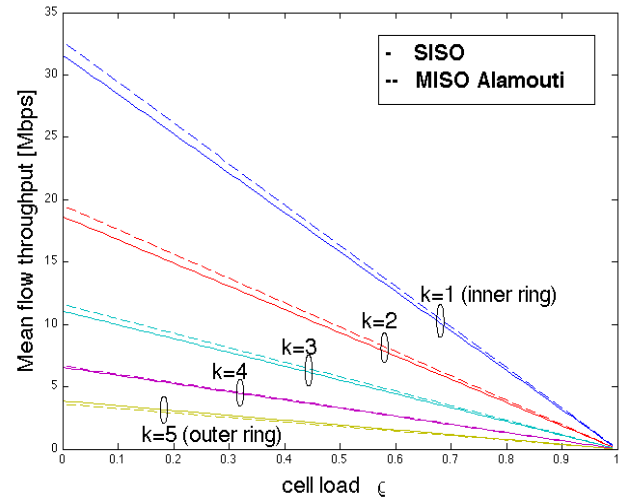

Fig. 1. Flow throughput vs cell load for SISO and $2 \times 1$ MISO Alamouti.

Fig. 2 shows a comparison between the $2 \times 2$ MIMO Alamouti with MRC system and the $2 \times 1$ MISO Alamouti system for the same values of the arrival rate. It can be seen that the MIMO Alamouti with MRC receiver system achieves a gain of about $4 \mathrm{Mbits} / \mathrm{s}$ in the inner ring compared to the MISO Alamouti system. The gain remains important in the outer ring.

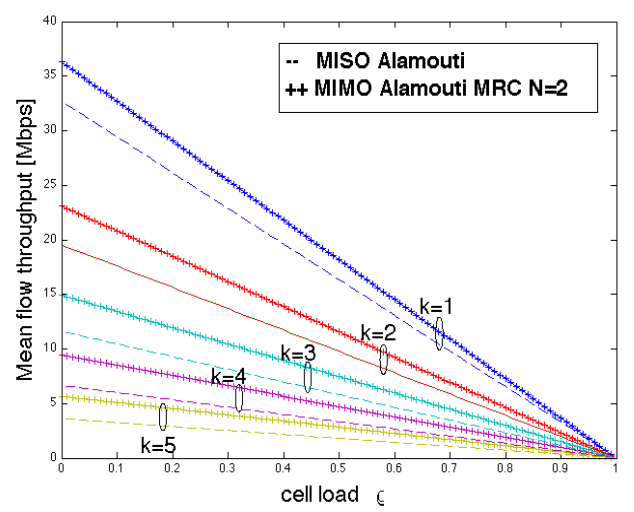

Fig. 2. Flow throughput vs cell load for $2 \times 1$ MISO Alamouti and $2 \times 2$ MIMO Alamouti MRC.

For SISO, MISO Alamouti and MIMO Alamouti with MRC, the cell capacities are respectively 6.17, 5.99 and $8.83 \mathrm{Mbps}$ (see (14)). This confirms the phenomenon observed in [10]: although Alamouti scheme greatly improves the outage probability at cell edge compared to SISO, it also provides relatively lower SINR at cell center. The two effects almost compensate and the cell capacities are similar. In contrast, the use of MRC significantly improves cell capacity.

\section{With Admission Control}

We now consider a minimum guaranteed rate $c_{m i n}=$ $0.5 \mathrm{Mbits} / \mathrm{s}$. The maximum number of active users in the cell can be obtained using (18) and is 12, 11 and 17 for SISO, MISO Alamouti and MIMO Alamouti with MRC resp. Based on this admission criterion, we compare the performance of our three systems. 
Fig. 3 illustrates a comparison between the flow throughput of the SISO system and the $2 \times 1$ Alamouti system as a function of the cell load. We notice that when $\rho \rightarrow 1$, the throughput of cell edge users tends to $c_{m i n}$. Then, cell capacities and maximum number of users being similar for the two systems, we obtain results in conformity with what has been observed without admission control.

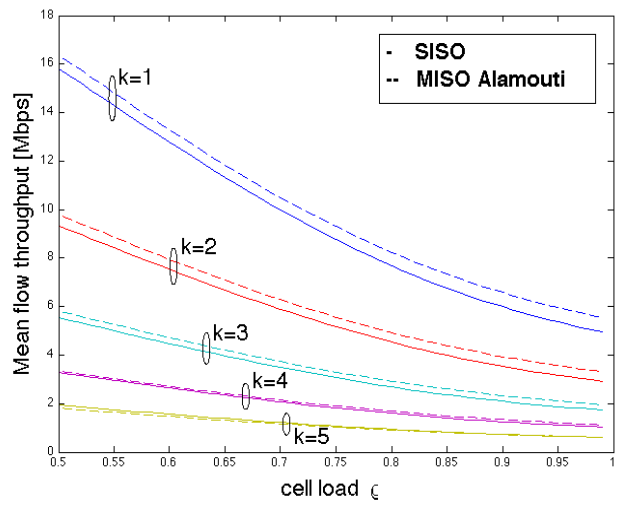

Fig. 3. Flow throughput vs cell load for SISO and $2 \times 1$ MISO Alamouti with admission control $\left(c_{\min }=0.5 \mathrm{Mbits} / \mathrm{s}\right)$.

Fig. 4 illustrates the comparison between MISO and MIMO. As expected, when the load is low, MIMO outperforms MISO in terms of flow throughput. It is however interesting to see that the flow throughput of MIMO users in inner rings fall below the throughput achieved by MISO users when the load is high.

From a mathematical point of view, it is not difficult to see that (19) decreases more rapidly when $m$ increases. More intuitively, the MIMO system accepts more users (17 instead of 11), so that the cell capacity (although higher) is divided in smaller pieces than with MISO. The loss is particularly visible for inner rings, i.e., users in the center of the cell.

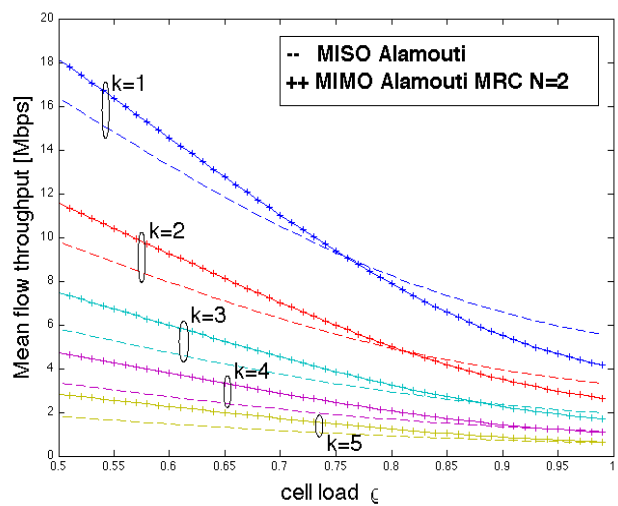

Fig. 4. Flow throughput vs cell load for $2 \times 1$ MISO Alamouti and $2 \times 2$ MIMO Alamouti MRC with admission control $\left(c_{\min }=0.5 \mathrm{Mbits} / \mathrm{s}\right)$.

\section{CONClusion}

In this paper, analytical expressions of the mean flow throughput have been derived for three systems: a SISO system, a MISO Alamouti transmission system and a MIMO Alamouti transmission with MRC receiver. The performance study has been derived assuming or not admission control. Performance results show that the MISO scheme does not bring enhancement over SISO in terms of flow throughput, although gains are obtained in terms of outage probability. Only the addition of MRC at the receiver, on top of the Alamouti scheme at the transmitter, brings significant improvements.

\section{REFERENCES}

[1] E. Telatar. Capacity of multi-antenna Gaussian channels. Technical report, AT \& T Bell Labs, June 1995.

[2] L. Zheng and D. Tse. Diversity and Multiplexing: A Fundamental Tradeoff in Multiple-Antenna Channels. IEEE Trans. on Inform. Theory, 49(5):1073-1096, May 2003.

[3] V. Tarokh, H. Jafarkhani, and A. R. Calderbank. SpaceTime Block Codes from Orthogonal Designs. IEEE Trans. on Inform. Theory, 45(5):1456-1467, July 1999.

[4] S. M. Alamouti. A simple transmit diversity technique for wireless communications. IEEE Journal on Selected Areas in Communications, 16(8):1451-1458, October 1998.

[5] A. Shah and A. Haimovich. Performance analysis of Maximum Ratio Combining and comparison with Optimum Combining for mobile radio communications with cochannel interference. IEEE Tran. on Veh. Tech., 49(4):1454-1463, July 2000.

[6] T. Bonald and A. Proutière. Wireless downlink data channels: User performance and cell dimensioning. In ACM Mobicom, Sept. 2003.

[7] T. Bonald, S. Borst, N. Hegde, and A. Proutière. Wireless data performance in multi-cell scenarios. In $A C M$ Sigmetrics, June 2004.

[8] S. Borst. User-level performance of channel-aware scheduling algorithms in wireless data networks. In IEEE Infocom, Mar. 2003.

[9] A. Papoulis. The Fourier Integral and Its Applications. McGraw-Hill, New York, NY, 1962.

[10] D. Ben Cheikh, J-M. Kelif, M. Coupechoux, and P. Godlewski. Outage probability in a multi-cellular network using alamouti scheme. IEEE Sarnoff Symposium 2010, 52(8):Princeton NJ, April 2010.

[11] WiMax System Evaluation Methodology. Wimax Forum, V2.1, July 2008.

[12] D. Ben Cheikh, J-M. Kelif, M. Coupechoux, and P. Godlewski. Multicellular alamouti scheme performance in rayleigh and shadow fading. Submitted to IEEE Trans. on Wireless Com.

[13] J-M. Kelif, M. Coupechoux, and P. Godlewski. Spatial outage probability for cellular networks. In IEEE Globecom 2007, Nov. 2007.

[14] C. Wengerter, J. Ohlhorst, and A.G.E. von Elbwart. Fairness and throughput analysis for generalized proportional fair frequency scheduling in ofdma. In IEEE VTC Spring, May 2005. 